\title{
NORMALIZERS OF SYSTEM NORMALIZERS
}

BY

\section{J. L. ALPERIN(1)}

1. The normalizers of the system normalizers are subgroups of some importance in the theory of solvable groups initiated by P. Hall [1-5]. For example, P. Hall observed [4] that a system normalizer was contained in the hypercenter of its normalizer. R. Carter analyzed extensively the class of solvable groups which have salf-normalizing system normalizers [6]. Furthermore, Carter, in a recent paper [7], has pointed out the importance of the question of the homomorphic invariance of the normalizers of the system normalizers. That is, if $H$ is the normalizer of a system normalizer $D$ of a solvable group $G$ and $N$ is a normal subgroup of $G$, is $H N / N$ the normalizer of the system normalizer $D N / N$ of $G / N$ ? This is equivalent with the following question $\left({ }^{2}\right)$ : If $D N / N$ is the normalizer of the system $T$ of $G / N$, is there a system $S$ of $G$ with normalizer $D$ such that $S$ is carried onto $T$ by the natural homomorphism of $G$ onto $G / N$ ?

For these reasons we have undertaken an investigation of the subgroups in the title of this paper. Our first result gives some more information about the embedding of a system normalizer in its normalizer.

THEOREM 1. Let $G$ be a solvable group of p-length one for all primes $p$. If $D$ is a system normalizer of $G$ then

$$
N(D)=D C(D) \text {. }
$$

In particular this means that the normalizer of $D$ induces only inner automorphisms on $D$. Furthermore, this theorem is false if the restrictions on $p$-length are relaxed. For example, if $G$ is the general linear group of two dimensions over the field of three elements, then $G$ is a nonsplit central extension of a cyclic group of order two by $S_{4}$, the symmetric group on four letters. The system normalizers of $G$ are elementary abelian of order four while their normalizers are dihedral groups of order eight.

The first theorem will be derived from the

THEOREM 2. Let $A$ be a group of automorphisms of the group G. Suppose that $A$ and $G$ have relatively prime orders and that at least one of the groups

Presented to the Society, April 19, 1963 under the title of System normalizers and Carter subgroups; received by the editors May 24, 1963.

(1) This research was partially supported by the Air Force Office of Scientific Research.

(2) Private communication from R. Carter. 
$A$ or $G$ is solvable $\left({ }^{3}\right)$. If $F$ is the subgroup of $G$ consisting of all elements left fixed by all automorphisms in $A$, then

$$
N_{G}(F)=F C_{G}(F) \text {. }
$$

This will be shown to be a corollary of the conjugacy statement of the SchurZassenhaus theorem [8, p. 162].

We shall now digress and state a recent result of J. Thompson which we will show follows from the above theorem.

Corollary. Let $P \times Q$ be a group of automorphisms of the p-group $G$ with $P$ a p-group and $Q$ a $p^{\prime}$-group. If every element of $G$ left fixed by $P$ is left fixed by $Q$ then $Q=1$.

Finally, we shall answer in the negative the question raised by Carter, which we mentioned above.

THEOREM 3. There exists a solvable group $G$ of nilpotent length three which has a system normalizer $D$, normal subgroup $N$ and maximal subgroup $M$ containing $D$ such that

(1) $N_{G / N}(D N / N) \neq N_{G}(D) N / N$,

(2) $D$ normalizes no system of $M$.

The second part of the statement of the theorem also answers a question raised by Carter [7]. Furthermore, the existence of this example seems to indicate many difficulties ahead. It stands directly in the path of attempts to prove certain conjectures by induction. In particular, Carter's "convergence" theorem [7, p. 549] may well be true in general, but an entirely new method of proof would be needed to show this.

The organization of the remainder of this paper is as follows. The rest of this section is devoted to a description of notation and relevant definitions, $\$ 2$ contains a proof of Theorem 2 , while $\S 3$ contains a proof of the corollary. $\S 4$ is devoted to Theorem 1 and the last section gives a description of the example of Theorem 3.

Let $G$ be a group with elements $x$ and $y$ and subgroups $G$ and $K$. We denote the commutator $(x, y)=x^{-1} y^{-1} x y$ and set $x^{y}=y^{-1} x y$. The subgroup generated by all commutators $(h, k)$ for $h \in H, k \in K$ will be written as $(H, K)$. The conjugate of $H$ by $x$ is $H^{x}$. The largest subgroup of $H$ which normalizes $K$ is $N_{H}(K)$ and the greatest subgroup of $H$ which centralizes $K$ is $C_{H}(K)$. If $H$ should contain $K$ then $(H: K)$ is the index of $K$ in $H$.

Now assume that this group $G$ is solvable. The Fitting subgroup $F$ of $G$ is the greatest normal nilpotent subgroup of $G$. If $G$ is nilpotent we say that $G$

(3) This is superfluous in view of the work of Feit and Thompson showing all groups of odd order are solvable. 
has nilpotent length one. Otherwise, we define the nilpotent length recursively, letting $G$ have nilpotent length one more than the nilpotent length of $G / F$. If $p$ is a prime and $S_{p}$ is a Sylow $p$-subgroup of $G$ we say that $G$ has $p$-length one provided there is a normal $p^{\prime}$-subgroup $N$ of $G$ such that $N S_{p}$ is a normal subgroup of $G$. Here, a $p^{\prime}$-subgroup is a subgroup of order prime to $p$.

The definitions of systems and system normalizers are contained in Hall's papers $[3 ; 4]$. The system normalizers form a conjugate class of nilpotent subgroups of any solvable group. Some of the most important properties of these subgroups are their "covering" and "avoidance" properties. This is described as follows: If $H / K$ is a chief factor of the solvable group $G$ and $D$ is a system normalizer of $G$ then each coset of $K$ in $H$ contains elements of $D(D$ covers $H / K)$ if $H / K$ is central in $G / K$ and no coset of $K$ in $H$, other than $K$ itself, contains any element of $D(D$ avoids $H / K)$ if $H / K$ is not central in $G / K$. Furthermore, if $N$ is a normal subgroup of $G$ then the system normalizers of $G / N$ are precisely the subgroups $D N / N$.

If $G$ is a $p$-group then we need to recall that every proper subgroup of $G$ is a proper subgroup of its normalizer. Also, if $A$ is a group of automorphisms of $G$ and $A$ leaves invariant a series of normal subgroups of $G$ and induces only the identity automorphisms on the successive factors, then $A$ is also a $p$-group. Such a series of subgroups is called a stability series for $A$.

2. The fixed-point theorem. We now turn to a proof of Theorem 2 and we let $A, F$ and $G$ be as described in the statement of that theorem. Since $A$ leaves fixed each element of $F$, it certainly leaves $F$ fixed as a subgroup. Therefore, each element of $A$ maps $N_{G}(F)$ onto itself. Thus, in order to prove the theorem, it is now clear that we may assume that $F$ is normal in $G$ and show that $G=F C_{G}(F)$.

We first note that if $N$ is a normal $A$-invariant subgroup of $G$ then $F N / N$ is the subgroup of $G / N$ of elements left fixed by the automorphisms induced on $G / N$ by the elements of $A$. In order to see this, we construct the splitting extension $G A$ of $G$ by $A$. An element of $G$ is in $F$ if and only if it lies in $N_{G}(A)$. Similarly, we form the splitting extension $(G / N) A$, so if $x \in G$ then $x N \in G / N$ is left fixed by the automorphisms induced by $A$ if and only if $x N \in N_{G / N}(A)$. However, this is true if and only if $x \in N_{G}(N A)$. In this case $A$ and $A^{x}$ are both complements of $N$ in $N A$. By the Schur-Zassenhaus theorem [8, p. 162], $A$ and $A^{x}$ are conjugate subgroups of $A N$. That is, there is $y \in N$ so that $A^{x y}=A$. Hence, $x y \in N_{G}(A)$ or $x y \in F$ so $x \in F N$ and the assertion at the beginning of the paragraph is proved.

In view of this, a proof of the theorem requires only a demonstration that $A$ induces only the identity automorphisms on $G / C_{G}(F)$. For then $G / C_{G}(F)$ $=F C_{G}(F) / C_{G}(F)$. However, suppose $x \in G, a \in A$ and $f \in F$. Then

$$
f^{x}=\left(f^{x}\right)^{a}=\left(f^{a}\right)^{x^{a}}=f^{x^{a}}
$$


so $x^{-1} x^{a} \in C_{G}(F)$ or $x C_{G}(F)=x^{a} C_{G}(F)$ for all $a \in A$ and $x \in G$. Therefore, the proof of Theorem 2 is complete.

In the case that $A$ is cyclic there is another proof of Theorem 2 available. Because this second proof is so different from the above one and in no way relies on the fundamental Schur-Zassenhaus theorem, we include this alternative method, in the hope that it may be of use elsewhere.

As above, we may assume that $F$ is a normal subgroup of $G$. Let $x$ be a generator of $A$ and suppose that $x$ has order $n$. Two paragraphs above we have shown that if $F$ is normal in $G, a \in A, g \in G$ then $(g, a) \in C_{G}(F)$. Thus, we need only show that each coset of $F$ in $G$ contains elements of the form $(g, a)$.

However, if $g$ and $h$ are in the same coset of $F$ in $G$ then $(x, g)=(x, h)$, since $x$ fixes each element of $F$. Therefore, to conclude this part of the proof, we need only show that if $g, h \in G$ and $F(x, g)=F(x, h)$ then $g$ and $h$ lie in the same coset of $F$ in $G$. If $(x, g) \in F(x, h)$ then $\left(g^{-1}\right)^{x} g F\left(h^{-1}\right)^{x} h=\left(h^{-1}\right)^{x} F h$ or $\left(h g^{-1}\right)^{x} \in F h g^{-1}$. Therefore, $\left(h g^{-1}\right)^{x}=f\left(h g^{-1}\right)$ for some $f \in F$. Hence, we next obtain $\left(h g^{-1}\right)^{x^{2}}$ $=f^{x}\left(h g^{-1}\right)^{x}=f^{2} h g^{-1}$ so finally

$$
h g^{-1}=f^{n} h g^{-1}
$$

and $f^{n}=1$. Since $G$ has order relatively prime to $n$ we deduce that $f=1$ so $\left(h g^{-1}\right)^{x}=h g^{-1}$. Thus $h g^{-1} \in F$ and $h \in F g$.

3. The corollary. Let $P, Q$ and $G$ be as in the statement of the corollary. We first form the splitting extension $G P$ of $G$ by $P$. Since $G$ and $P$ are $p$-groups so will $G P$ also be a $p$-group. Since $P$ and $Q$ generate their direct product we may consider $Q$ as a group of automorphisms of $G P$. The subgroup of elements of $G P$ left fixed by $Q$ will then be a product $F P$ of $P$ and the subgroup $F$ of $G$ of elements left fixed by $Q$. The theorem now gives us that

$$
N_{G P}(F P)=F P C_{G P}(F P) \text {. }
$$

However, $C_{G P}(F P)$ is a subgroup of $C_{G P}(P)$ and the hypothesis of the corollary is no more than the assertion that $C_{G}(P)$ is a subgroup of $F$. Thus, $C_{G P}(P)$ is a subgroup of $F P$ so the above application of the theorem gives

$$
N_{G P}(F P)=F P \text {. }
$$

But $G P$ is a $p$-group so this implies that $F P=G P$ or $F=G$ and finally $Q=1$.

4. The central product theorem. Let $G$ be a solvable group of $p$-length one, with system normalizer $D$, Sylow $p$-subgroup $D_{p}$ of $D$ and Sylow $p$-subgroup $S_{p}$ of $G$ containing $D_{p}$. As a first step toward proving Theorem 1 we shall show that a similar statement holds for the embedding of $D_{p}$ in $S_{p}$, namely,

$$
N_{S_{n}}\left(D_{p}\right)=D_{p} C_{S_{n}}\left(D_{p}\right) \text {. }
$$


Furthermore, in proving this, we shall proceed by induction on the order of $G$.

Let $N$ be the largest normal subgroup of $G$ of order prime to $p$ and suppose $N \neq 1$. Let $x \in N_{S_{n}}\left(D_{p}\right)$ so $x N$ normalizes $D_{p} N / N$ in $G / N$. Since $N \neq 1$, we may apply the induction hypothesis to $G / N$, so that we have $x=y z$ where $y \in D_{p} N$ and $z \in S_{p} N$ with $z N$ centralizing $D_{p} N / N$ in $S_{p} N / N$. Thus we may let $y=u m$, $z=v n$ where $u \in D_{p}, v \in S_{p}, m, n \in N$ and $v$ centralizing $D_{p}$ modulo $N$. Therefore, $x=u m v n=u v m^{v} n$ so $x=u v$ and $m^{\prime \prime} n=1$. To conclude the proof in the case when $N \neq 1$ we need only see that $v \in C_{S_{n}}\left(D_{p}\right)$. But if $d \in D_{p}$ then $(d, v) \in N \cap D_{p}=1$ and we are done in this case.

However, if $N=1$ then $S_{p}$ is a normal subgroup of $G$ because $G$ has $p$-length one. We now let $A$ be a complement of $S_{p}$ in $G$. The subgroup $A$ induces a group of automorphisms on $S_{p}$ and a direct application of the theorem now disposes of this case.

As a second step toward the proof of Theorem 1 we shall show that, with the notation above,

$$
N_{G}\left(D_{p}\right)=D_{p} C_{G}\left(D_{p}\right)
$$

Let $G=G_{0}>G_{1}>\cdots>G_{n}=1$ be a chief series for $G$. Let $K_{i}=D_{p} \cap G_{i}$ for $i=0,1, \cdots, n$. If $x \in N_{G}\left(D_{p}\right)$ then $x$ normalizes each subgroup $K_{i}$ since $x$ certainly normalizes the subgroups $G_{i}$. By the avoidance property of system normalizers, we will have $K_{i}=K_{i+1}$ if $G_{i} / G_{i+1}$ is a noncentral chief factor of $G$. Therefore, if $K_{i} \neq K_{i+1}, x$ will centralize $G_{i} / G_{i+1}$ and so will centralize $K_{i} / K_{i+1}$. Hence, the group of automorphisms $N_{G}\left(D_{p}\right) / C_{G}\left(D_{p}\right)$ of $D_{p}$ stabilizes the series of subgroups $D_{p}=K_{0} \geqq K_{1} \geqq \cdots \geqq K_{n}=1$. Therefore, since $D_{p}$ is a $p$-group, $N_{G}\left(D_{p}\right) / C_{G}\left(D_{p}\right)$ is a $p$-group. The desired conclusion now follows from the assertion proved at the beginning of this section. Indeed, $N_{G}\left(D_{p}\right)=N_{S_{n}}\left(D_{p}\right) H$ where $H$ is a $p^{\prime}$-subgroup of $N_{G}\left(D_{p}\right)$, so $H$ centralizes $D_{p}$ and $N_{S}\left(D_{p}\right)=D_{p} C_{S}\left(D_{p}\right)$ and we have $N_{G}\left(D_{p}\right)=D_{p} C_{G}\left(D_{p}\right)$.

We now proceed with the proof of Theorem 1 . Let $p_{1}, p_{2}, \cdots, p_{n}$ be the distinct primes dividing the order of $G$ and assume that $G$ has $p_{i}$-length one for all $p_{i}, i=1,2, \cdots, n$. Let $D=D_{p_{1}} \times D_{p_{2}} \times \cdots \times D_{p_{n}}$ where $D_{p_{i}}$ is the Sylow $p_{i}$-subgroup of $D$. Let $x \in N_{G}(D)$. Thus $x \in N_{G}\left(D_{p_{1}}\right)$ because $D_{p_{1}}$ is a characteristic subgroup of $D$. Therefore, we may write $x=y_{1} x_{1}$ where $y_{1} \in D_{p_{1}}$ and $x_{1} \in C_{G}\left(D_{p_{1}}\right)$. Thus $x_{1}=y_{1}^{-1} x \in N_{G}(D)$ since $y_{1} \in D$. Similarly, we may express $x_{1}=y_{2} x_{2}$ where $y_{2} \in D_{p_{2}}$ and $x_{2} \in C_{G}\left(D_{p_{2}}\right)$. However, $x_{2}=y_{2}^{1-} x_{1} \in C_{G}\left(D_{p_{1}}\right)$ since $y_{2} \in D_{p_{2}}$ and $x_{1} \in C_{G}\left(D_{p}\right)$. Thus $x_{2} \in C_{G}\left(D_{p_{1}} \times D_{p_{2}}\right)$. Continuing in this way we finally obtain the expression

$$
x=y_{1} y_{2} \cdots y_{n} x_{n}
$$

where $y_{i} \in D_{p_{i}}, i=1,2, \cdots, n$ and $x_{n} \in C_{G}\left(D_{p_{1}} \times \cdots \times D_{p_{n}}\right)=C_{G}(D)$. This completes the proof. 
5. The example. We shall now construct and analyze the group described in Theorem 3. As a first step, we let $p$ be a prime other than two or three. Let $P$ be the largest group on three generators which is of exponent $p$ and nilpotent of class two. Then $P$ has generators $u_{1}, u_{2}$ and $u_{3}$ subject to the defining relations

$$
u_{1}^{p}=u_{2}^{p}=u_{3}^{p}=\left(u_{i}, u_{j}, u_{k}\right)=1 .
$$

If we denote $u_{i_{j}}=\left(u_{i}, u_{j}\right)$ for $1 \leqq i<j \leqq 3$ then $u_{i j}$ has order $p$ and every element of $P$ has a unique representation in the form

$$
u_{1}^{a_{1}} u_{2}^{a_{2}} u_{3}^{a_{3}} u_{12}^{a_{12}} u_{13}^{a_{13}} u_{23}^{a_{23}}
$$

where the $a_{i}$ and $a_{j k}$ are integers with $0 \leqq a_{i}, a_{j k}<p$. The center and derived group of $P$ coincide and consist of the elements which have $a_{1}=a_{2}=a_{3}=0$ when expressed in this way.

The symmetric group $S_{3}$ on three letters permutes the elements $u_{1}, u_{2}$ and $u_{3}$ and these three permutations induce a group of automorphisms of $P$ isomorphic with $S_{3}$. Let $x$ be the automorphism which interchanges $u_{1}$ and $u_{2}$ and leaves $u_{3}$ fixed and let $y$ be the automorphism which maps $u_{1}$ to $u_{2}, u_{2}$ to $u_{3}$ and $u_{3}$ to $u_{1}$. We now let $G$ be the splitting extension of $P$ by $S_{3}$ with $S_{3}$ acting as a group of automorphisms as just described.

Hence, the subgroups $X, Y$ and $P$, where $X$ and $Y$ are the cyclic subgroups generated by $x$ and $y$ respectively, are a Sylow 2-subgroup, Sylow 3-subgroup and a Sylow p-subgroup of $G$. Furthermore, these three subgroups and their products form a Sylow system of $G$. If we let $D$ be the normalizer of this system, then the Sylow 2-subgroup $D_{2}$ of $D$ is the largest subgroup of $X$ normalizing $Y P$ and so is $X$ itself. Similarly, the Sylow 3-subgroup $D_{3}$ of $G$ is the largest subgroup of $Y$ normalizing $X P$. However, $(x, y)=\left(y^{-1}\right)^{x} y=y^{2} \notin X P$, so $D_{3}=1$. The Sylow $p$-subgroup $D_{p}$ of $D$ consists of those elements of $P$ left fixed by the automorphisms induced by $X Y$. However, if $u$ is an element of $P$, written in the canonical form given above, then $u$ is sent by $x$ into the element

$$
u_{2}^{a_{1}} u_{1}^{a_{2}} u_{3}^{a_{3}} u_{12}^{-a_{12}} u_{23}^{a_{13}} u_{13}^{a_{23}}=u_{1}^{a_{2}} u_{2}^{a_{1}} u_{3}^{a_{3}} u_{12}^{-a_{12}-a_{1} a_{2}} u_{13}^{a_{23}} u_{23}^{a_{13}}
$$

so if $u$ is centralized by $x$ then $a_{1} a_{2}+a_{12} \equiv 0, a_{13}=a_{23}$ (congruences modulo $p$ ). Similarly, $u$ is sent by $y$ into

$$
u_{2}^{a_{1}} u_{3}^{a_{2}} u_{1}^{a_{3}} u_{23}^{a_{12}} u_{12}^{-a_{13}} u_{13}^{-a_{23}}=u_{1}^{a_{3}} u_{2}^{a_{1}} u_{3}^{a_{2}} u_{12}^{-a_{12} a_{3}-a_{13}} u_{13}^{-a_{23}-a_{2} a_{3}} u_{23}^{-a_{23}}
$$

so if $u$ is centralized by $y$ then

$$
a_{1}=a_{2}=a_{3}, \quad a_{23} \equiv-a_{23}, \quad a_{13}+a_{1} a_{3} \equiv a_{23}+a_{2} a_{3} \equiv 0 .
$$


Therefore, it is easy to see that $D_{p}$ is cyclic and of order $p$ and generated by

$$
d=u_{1} u_{2} u_{3} u_{12}^{-1 / 2} u_{13}^{-1 / 2} u_{23}^{-1 / 2} .
$$

Consequently, $D$ is the subgroup of order $2 p$ generated by $x$ and $d$.

The normalizer $N(D)$ of $D$ is easy to calculate. Indeed, any element of $g \in G$ which normalizes $D$ must normalize $D_{2}=X$ and so commute with $x$. Furthermore $g$ must normalize the subgroup $D_{p}$. In fact $N(D)$ is the subgroup of order $2 p^{2}$ generated by $x, d$ and $u_{13} u_{23}$.

However, if we let $N$ be the derived group of $P$, then $N$ is a normal subgroup of $G$. Furthermore, the element $u_{1} u_{2} N$ of $G / N$ normalizes $D M / N$ but no element of $G$ in the coset $u_{1} u_{2} N$ lies in $N(D)$. Therefore, $N$ has the properties required by the theorem.

Furthermore, if we let $M$ be the subgroup of $G$ generated by $X Y$ together with $u_{1} u_{2} u_{3}$ and $N$, then it is easy to see that $M$ is a maximal subgroup of $G$ of index $p^{2}$ in $G$. Indeed, any coset of $M$ in $G$ contains an element of the form $u_{1}^{a} u_{2}^{b}$ and $M$ and any such element generate $G$. Let $v=u_{1} u_{2} u_{12}^{-1 / 2}$ so $v$ centralizes $X$ and let $D_{1}=D^{v}$. Thus, $D_{1}$ is a system normalizer of $G$ and is contained in $M$. We shall show that $D_{1}$ normalizes no system of $M$ by proving that $D_{1}$ normalizes no Sylow 3-subgroup of $M$.

To accomplish this we shall first determine the Sylow 3-subgroups of $G$ normalized by $D$. We note that any two systems of $G$ normalized by $D$ are conjugate by an element of $N(D)$. For, if $S$ and $T$ are two systems normalized by $D$ they are certainly conjugate in $G$, say by an element $g$. Thus $D=N(S)$ so $D^{g}=N\left(S^{g}\right)=N(T)$. But $N(T)=D$ so $D=D^{g}$ and $g \in N(D)$. Now, if $W$ is a Sylow 3-subgroup of $G$ normalized by $D$ then $X$ normalizes $W$ so that $S, W$ and $P$ and their products form a Sylow system of $G$ normalized by $D$. Thus, in order to find the Sylow 3-subgroups of $G$ normalized by $D$ we need only determine the Sylow 3-subgroups of the systems normalized by $D$. But, as we have just seen above, these are the conjugates of $Y$ by the elements of $N(D)$. Thus, the conjugates of $Y$ normalized by $D$ are precisely the subgroups $Y^{w^{a}}$ when $w=u_{13} u_{23}$ and $a=0,1, \cdots, p-1$.

To conclude, we recall that $D_{1}=D^{v}$ so therefore the Sylow 3-subgroups of $G$ normalized by $D_{1}$ are the subgroups $Y^{w^{a} v_{v}}$. However, it is easy to check that none of these Sylow groups is contained in $M$ so $D_{1}$ is contained in $M$ and normalizes no system of $M$. Hence, $D$ is contained in $M^{v^{-1}}=M_{1}$ and normalizes no system of $M_{1}$. This completes the proof of Theorem 3 .

Concluding Remarks. The above results suggest two directions for possible further investigations. The first would be a search for an analog of Theorem 1 for groups of arbitrary $p$-length. The second direction, and this is vague, would be an attempt to try and get around all the difficulties presented by Theorem 3 . Progress in either direction would certainly bring us deeper knowledge of the structure of solvable groups. 


\section{REFERENCES}

1. P. Hall, A note on soluble groups, J. London Math. Soc. 3 (1928), 98-105.

2. - A characteristic property of soluble groups, J. London Math. Soc. 12 (1937), 198-200.

3. - On the Sylow systems of a soluble group, Proc. London Math. Soc. (2) 43 (1937), 316-323.

4. - On the system normalizers of a soluble group, Proc. London Math. Soc. (2) 43 (1937), 507-528.

5. - The construction of soluble groups, J. Reine Angew. Math. 182 (1940), 206-214.

6. R. W. Carter, On a class of finite soluble groups, Proc. London Math. Soc. (3) 9 (1959), 623-640.

7. - Nilpotent self-normalizing subgroups and system normalizers, Proc. London Math. Soc. (3) 12 (1962), 535-563.

8. H. J. Zassenhaus, The theory of groups, 2nd ed., Chelsea, New York, 1958.

Massachusetts Institute of Technology,

CAmbridge, MassachusetTs 